\title{
A Test Collection for Interactive Lifelog Retrieval
}

\author{
Cathal Gurrin ${ }^{1}$, Klaus Schoeffmann ${ }^{2}$, Hideo Joho ${ }^{3}$, Bernd Munzer ${ }^{2}$, Rami \\ Albatal $^{1}$, Frank Hopfgartner ${ }^{4}$, Liting Zhou ${ }^{1}$, and Duc-Tien Dang-Nguyen ${ }^{1,5}$ \\ 1 Insight Centre for Data Analytics, Dublin City University, Ireland \\ 2 Klagenfurt University, Austria \\ 3 University of Tsukuba, Japan \\ 4 University of Sheffield, UK \\ 5 University of Bergen, Norway
}

\begin{abstract}
There is a long history of repeatable and comparable evaluation in Information Retrieval (IR). However, thus far, no shared test collection exists that has been designed to support interactive lifelog retrieval. In this paper we introduce the LSC2018 collection, that is designed to evaluate the performance of interactive retrieval systems. We describe the features of the dataset and we report on the outcome of the first Lifelog Search Challenge (LSC), which used the dataset in an interactive competition at ACM ICMR 2018.
\end{abstract}

Keywords: interactive retrieval, lifelogging, comparative evaluation, test collection, multimodal dataset

\section{Introduction}

Dodge and Kitchin [6] refer to lifelogging as 'a form of pervasive computing, consisting of a unified digital record of the totality of an individual's experiences, captured multimodally through digital sensors and stored permanently as a personal multimedia archive'. Technological progress and cheaper sensors has enabled people to capture such digital troves of life experiences automatically and continuously with ease and efficiency. Ongoing research is constantly optimising the user experience on these systems. A lifelog, according to the definition of Dodge and Kitchin, should consist of rich media data that captures, in so far as possible, a digital trace of the totality of an individual's experience. Such a lifelog should be a rich media archive of personal contextual data, which includes various forms of biometric data, physical activity data, wearable media, as well as data on the information creation and consumption of the individual.

In the spirit of Memex [2], it is our conjecture that a lifelog, if it is to be useful to the individual, must be 'continuously extended, it must be stored, and above all it must be consulted'. Such lifelog consultation is likely to require both ad-hoc and interactive retrieval mechanisms to support the variety of lifelog usecases, as suggested in [20]. While we note significant efforts being made through 
various vehicles, such as NTCIR [10] and ImageCLEF [4], to support off-line adhoc search tasks, by the release of a first generation of lifelog test collection, until now, there was no dedicated benchmarking effort for interactive lifelog search, nor is there a test collection designed to support such benchmarking.

As reported in [5], the design and creation of a reusable lifelog test collection for any form of retrieval experimentation is not trivial. Jones and Teeven [12], in the context of personal information management (PIM), state that "the design of shared test collections for PIM evaluation requires some creative thinking, because such collections must differ from more traditional shared test collections". In this paper, we report on the first such test collection, the LSC2018 collection, which was designed to support interactive lifelog search and was first used in the live LSC 2018 (Lifelog Search Challenge) competition at ACM ICMR 2018. We describe the test collection, motivate its development and report on the six experimental interactive retrieval systems that took part in the LSC and utilised the test collection. Hence, the contributions of this paper are as follows:

- A description of a new test collection that can be used to support interactive lifelog search, with associated details on how to access the collection.

- A review of the first interactive lifelog search systems that took part in the LSC 2018 workshop at ACM ICMR 2018.

- The introduction of a new type of query for interactive retrieval that is designed to become progressively easier during a time-limited interactive search competition.

\section{Related Collections \& Evaluation Forums}

Collecting and organising lifelog data is clearly different from conventional data in many aspects. In 2010, Sellen and Whittaker [20] argued that rather than trying to capture everything, the so called "total capture", lifelog system design should focus on the psychological basis of human memory to reliably organise and search on personal life archives. The technical challenges arising from either focused or total capture, include the indexing and the organisation of heterogeneous media, such as image, audio, video and sensor data, along with the development of a suite of interface tools to support access and retrieval. Many researchers have proposed lifelog retrieval systems, such as the eLifeLog system from Kim and Giunchiglia [14] and demonstrated the potential of their system on an archive of rich, multi-modal and event-based annotated data. However, in the majority of cases, such multimodal datasets were not released to the community.

In the last three years, large volumes of multimodal lifelog data have been gathered from several lifeloggers and released as part of the NTCIR collaborative benchmarking workshop series [13] in dedicated Lifelog tracks/tasks. To the best of our knowledge, as of October 2018, these collections (such as the NTCIR-12 Lifelog collection [8] and the NTCIR-13 Lifelog collection [10]) are the largest (in terms of number of days and the size of the collection) and richest (in terms of types of information) collections on lifelogging ever shared. These collections are summarised in Table 1. 
Table 1. Statistics of the NTCIR Collections.

\begin{tabular}{l|l|l} 
& NTCIR-12 & NTCIR-13 \\
\hline Number of Lifeloggers & $\mathbf{3}$ & $\mathbf{2}$ \\
Number of days & $\mathbf{8 7}$ & $\mathbf{9 0}$ \\
Size of the Collection (GB) & $\mathbf{1 8 . 1 8}$ & $\mathbf{2 6 . 6}$ \\
Size of the Collection (Images) & $\mathbf{8 8 , 1 2 4}$ & $\mathbf{1 1 4 , 5 4 7}$ \\
Size of the Collection (Locations) & $\mathbf{1 3 0}$ & $\mathbf{1 3 8}$
\end{tabular}

Based on the collections from NTCIR-12 and NTCIR-13, rigorous comparative benchmarking initiatives have been organised: the NTCIR 12 - Lifelog [9], and ImageCLEFlifelog2017 [3] exploited the NTCIR-12 collection and NTCIR-13 Lifelog 2 [10], ImageCLEFlifelog2018 [4] were proposed based on the NTCIR-13 collection. Typically, for each benchmarking initiative, based on the collection employed, several tasks were introduced which aim to advance the state-of-theart research in lifelogging as an application of information retrieval.

Concerning only-visual collections of lifelog data, there have been a small number released in the last five years. For example, the UT Ego Dataset [15] contains four (3-5 hour long) videos captured from head-mounted cameras, captured in a natural, uncontrolled setting; the Barcelona E-Dub dataset [23] contains a total of 18,735 images captured by 7 different users during overall 20 days; and the Multimodal Egocentric Activity Dataset [22] contains 20 distinct lifelogging activities (10 videos for each activity and each video lasts 15 seconds) performed by different human subjects.

Related to interactive information retrieval efforts and datasets, the Video Browser Showdown (VBS) is an international video search competition with the goal to evaluate the state-of-the-art performance of interactive video retrieval systems on a large shared dataset [16] of video data. It is held as a special session at the International Conference on Multimedia Modeling (MMM), annually since 2012. In this competition several teams work in front of a shared screen and try to solve a given set of Known-Item Search (KIS) and Ad-Hoc Video Search (AVS) tasks as fast as possible, where the tasks are selected randomly on-site. The difference between the task types is that a KIS task would seek a specific single target clip in the entire collection - described either as a shown clip or as a textual description, while an AVS task would requite all shots belonging to a particular topic (e.g., "find all shots with cars on the street") to be found. The tasks are issued and scored by the VBS server, to which all teams are connected to. For scoring, the server evaluates the search time and correctness of each submission and computes a score for the team. In general, the scoring is higher the faster a correct submission has been sent (and the less false submissions were sent before) - for AVS tasks, however, it will also matter how many different instances were found and from how many different videos they are coming. The whole competition consists of expert and novice sessions, where in the latter kind volunteers from the conference audience work with the tools of the experts. The final score is computed as an average over all sessions (expert KIS visual, expert KIS textual, expert AVS, novice KIS visual, novice AVS). In VBS2018, 
the IACC. 3 dataset has been used for the competition, which consists of 600 hours of content and about 300,000 shots. For each session the participants had 5 minutes to solve an AVS or KIS task.

\section{Justification for a New Test Collection}

At the time of preparing the LSC workshop, both the NTCIR-12 and NTCIR13 were the only readily available large-scale lifelog test collections. While, in theory, it was possible for any collection (large or small) to be employed for interactive retrieval, our conjecture was that in order to encourage participation of researchers from a variety of fields (MMIR, HCI, etc), that we needed to provide a reasonably sized collection that contained real-world, multi-modal lifelog data, along with sufficient metadata, so as to reduce the barriers to entry for non-computer-vision researchers, who had heretofore been the main users of lifelog collections.

Both of the existing NTCIR test collections were (relatively) large lifelog collections with limited metadata and 24-48 ad-hoc topics with relevance judgements. While additional topics could have been generated for these test collections, it was decided that a test collection was needed with richer metadata, which would facilitate additional facets of retrieval to be integrated into an interactive querying engine. Hence, the LSC test collection was created, which was a subset of the NTCIR-13 collection, but with additional metadata, namely complete biometric data 24/7, detailed anonymised location logs, and the new source of informational data including information consumed and created on computer devices.

\section{LSC 2018, a Test Collection for Interactive Lifelog Search}

The conventional structure of a test collection requires three components, namely: 1) a collection of domain-representative documents, 2) a set of queries (called topics) that are representative of the domain of application, and 3) a set of relevance judgements that map topics to documents. The LSC test collection contains all three components, and since it was based on a subset of the NTCIR-13 collection, was it developed according to the same process outlined in [10].

\subsection{Requirements for the Test Collection}

Prior to generating the test collection we defined requirements for the collection based on our experiences of running the NTCIR 12 \& 13 Lifelog tasks [9], [10] and relevant literature concerning lifelogging and human memory, such as [20]. To summarise, these requirements were:

- be a valid test collect of real-world lifelog data and information needs from a wide variety of sensors. 
- that appropriate metadata be included with the collection so as to reduce the barriers to use of the collection

- all user identifiable data must be removed from the collection.

The NTCIR-13 Lifelog test collection was created according to these requirements and included all day data gathering by volunteer lifeloggers using multiple devices. All data was then temporally aligned to UTC time (Coordinated Universal Time) and the data was filtered by firstly the lifelogger themselves and then by a trusted expert. This data was then enhanced by the addition of various forms of metadata before all user identifiable content was removed and the collection made available.

For the subsequent creation of the LSC test collection, 27 days of the NTCIR13 Lifelog data from one lifelogger was extracted, due to the presence of the richest lifelog data from this period of time. Both GPS location (with work and home removed) and additional computer content access and creation data was added to the collection. We now describe the collection in detail.

\subsection{Test Collection Description}

Data Although the collection is based on the NTCIR-13 Lifelog collection, there are a number of additions, as described above, so we summarise the collection thus:

- Multimedia Content. Wearable camera images were gathered using a Narrative Clip 2 wearable camera capturing about two images per minute and worn from breakfast to sleep, at a resolution of 1024 x 768, with faces blurred. Examples are shown in Figure 4.2. Accompanying this image data is a timestamped record of music listening activities sourced from Last.FM.

- Biometric Data. Using the Basis smartwatch, the lifeloggers gathered $24 \times 7$ heart rate, galvanic skin response, calorie burn and steps, on a per-minute basis. In addition, daily blood pressure and blood glucose levels were recorded every morning before breakfast and weekly cholesterol and uric acid levels were recorded.

- Human Activity Data. The daily activities of the lifeloggers were captured on a per-minute basis, in terms of the semantic locations visited, physical activities (e.g. walking, running, standing) along with a time-stamped diet$\log$ of all food consumed drinks taken, and a location record for every minute. An example of the locations is shown in Figure 4.2.

- Information Activities Data. Using the Loggerman app, the information creation and consumption activities were provided, which were organised into blacklist-filtered, sorted, document vectors representing every minute.

In order to make the collection more suitable for interactive retrieval, the wearable camera images were annotated with the outputs of a semantic concept detector from Microsoft cognitive services (computer vision API) [21], which provided high-quality annotations of visual concepts from the visual lifelog data. 

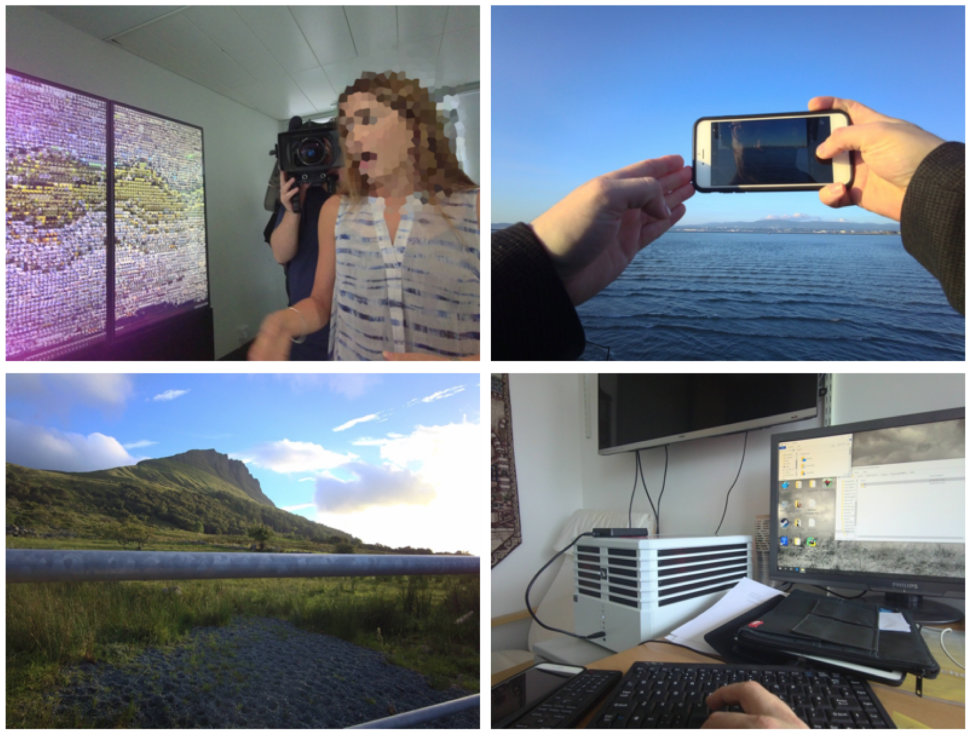

Fig. 1. Examples of Wearable Camera Images from the Test Collection

Topics \& Relevance Judgements Being a test collection designed for interactive retrieval, the topics selected to facilitate interactive retrieval and competitive benchmarking in a live setting. Hence we introduced a new type of interactive topic that was designed around the concept of temporal enhanced query descriptions. A topic was created based on the lifelogger selecting a memorable and interesting event that had occurred during the time period covered by the test collection. The guidance given to the lifelogger was that the event should

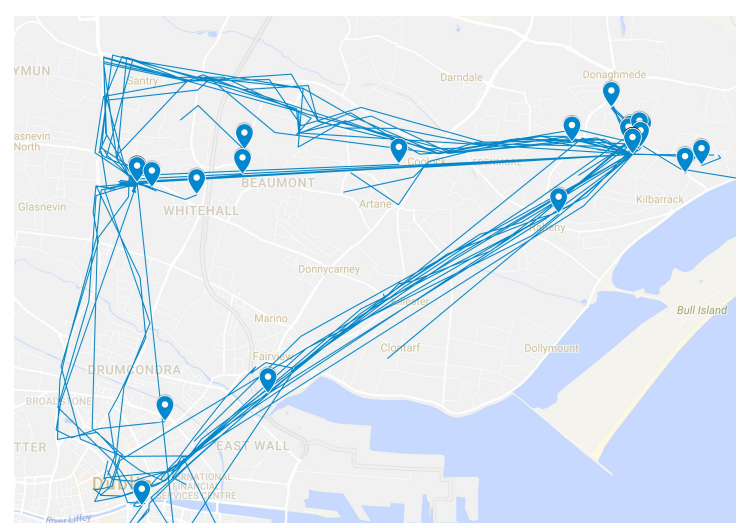

Fig. 2. Examples of the Locations from the Test Collection. 
Table 2. Statistics of LSC 2018 Lifelog Data

\begin{tabular}{l|l}
\hline Number of Lifeloggers & $\mathbf{1}$ \\
Number of Days & $\mathbf{2 7}$ \\
Size of the Collection (GB) & $\mathbf{9 . 4 0}$ \\
Number of Images & $\mathbf{4 1 , 6 8 1}$ \\
Number of Locations & $\mathbf{7 2}$ \\
Number of Development Topics & $\mathbf{6}$ \\
Number of Expert Topics & $\mathbf{6}$ \\
Number of Novice Topics & $\mathbf{1 2}$ \\
Number of unique concepts & $\mathbf{4 9 0}$ \\
\hline
\end{tabular}

ideally only occur either once or a few times in the collection. Each topic was represented by an information need that described the user context in detail, including locations, days of the week and visual elements of the image(s) that matched the topic. The rationale was that a user with an interactive system that included a range of facets would be able to quickly locate content of interest. However, since the topics were to be employed in a live search competition, the topics were designed to be temporally extended through six iterations, with each iteration lasting for 30 seconds and providing increasing levels of contextual data to assist the searcher. With six iterations in total, this resulted in a total time allocation of three minutes per topic. An example of a topic is shown below.

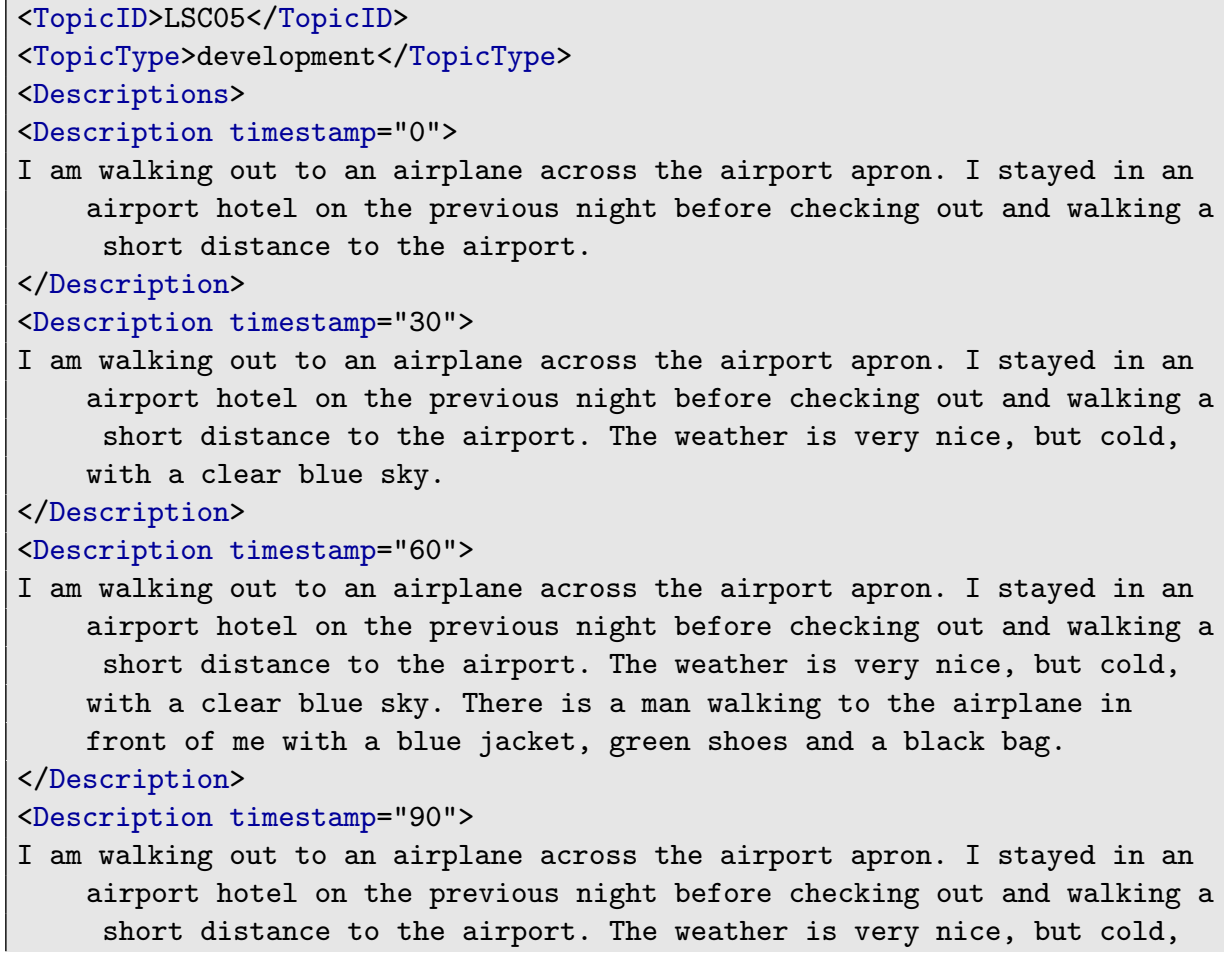




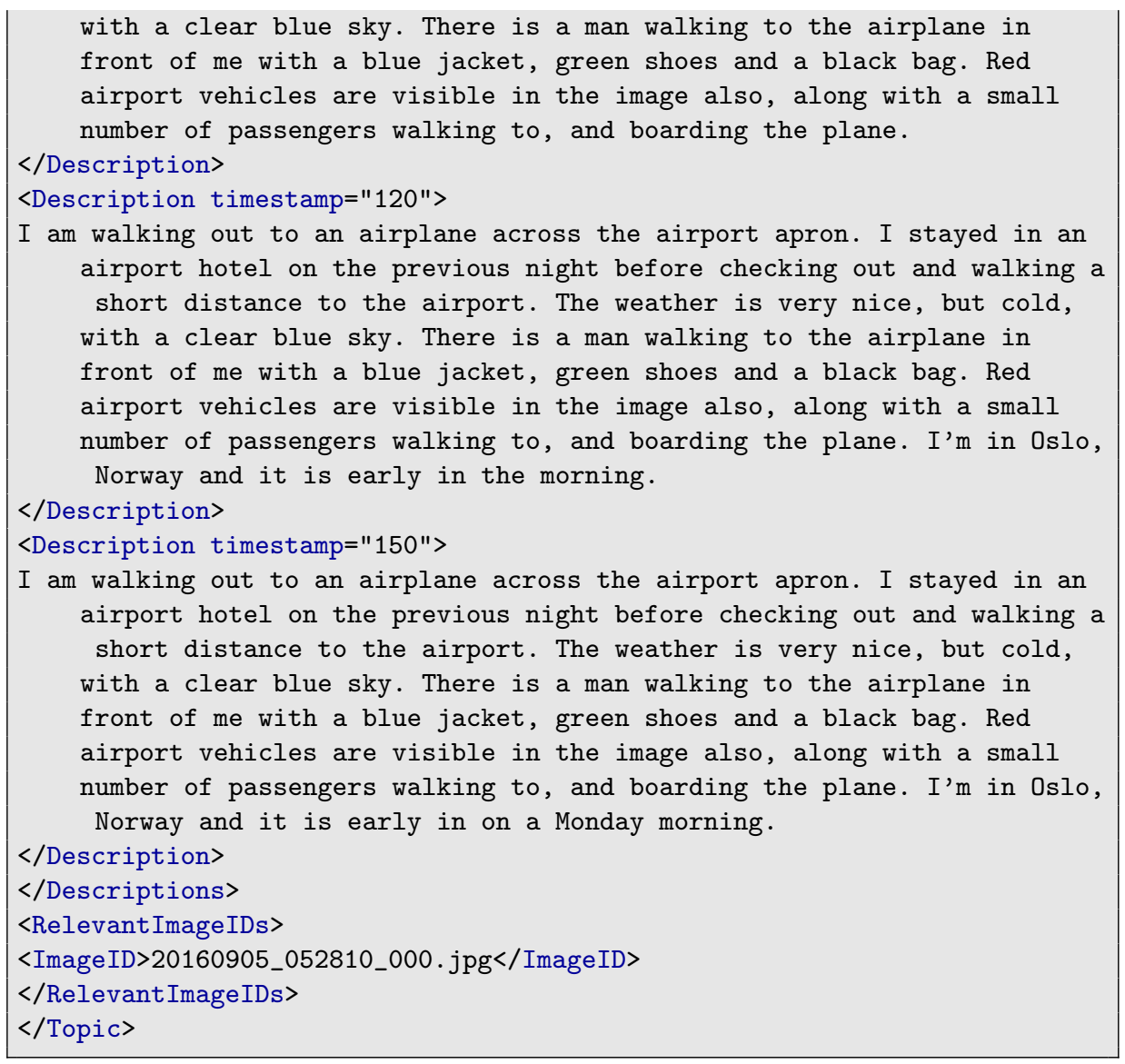

There were three types of topic in the test collection. Six development topics (as above), six test topics for experts (system developers) for the search challenge, and twelve test topics for novice users, who were not knowledgeable about the collection or how the systems worked. All three types of topic had the same structure.

Associated with each topic were the relevance judgements generated manually by the lifelogger. As stated, there could be one or more relevant items in the collection, where relevant items could span multiple separate events or happenings. In this case, if a user of an interactive system found any one of the relevant items from any event, then the search is deemed to be successful. For the LSC collection, an item was assumed to be an image from the wearable camera.

\subsection{Collection Applications}

The LSC dataset was primarily developed to support comparative benchmarking of interactive lifelog retrieval systems. It was designed to be easy to employ, as well as to provide multi-level challenging topics. In addition to this primary 
application at LSC 2018, we also note that, due to the richness of the contextual data it provides, that the collection is already being employed by additional researchers to support:

- User Context Modelling, by identifying the tasks of daily life and modelling a user's life activities as a sequence of tasks.

- Hybrid Data Modelling, to develop various event detectors for daily life, such as fall event detection, or important moment detectors.

- Personal Data Engines, to provide prototype retrieval systems over personal data archives.

It is our conjecture that this collection can be employed for many other aspects of multimedia information retrieval, such as lifestyle activity detection, real-world task identification, multimodal retrieval systems, and so on.

\subsection{Collection Limitations}

While the LSC collection is the first interactive lifelog collection, there are a number of limitations that we wish to point out:

- The main limitation is the size of the collection, which is only 27 days of data. This time period was chosen because it gave the optimal trade-off between richness of gathered data and the duration of the collection. Ideally, this should be a longitudinal collection extending to several months at least. Small collections for interactive search can become familiar to expert searchers who can use this extra knowledge to assist in the search process.

- Another limitation of the collection is that the multimodal lifelog data does not include media such as contextual audio or non-written communications.

- A third limitation is the fact that the collection has been anonymised via a process that blurs faces and makes screens illegible. This was a necessary part of the data release process, but it restricts the type of queries that can be used with the collection.

\section{Employing the Collection at the LSC}

The LSC dataset was employed for the Lifelog Search Challenge (LSC), at ACM ICMR in June 2018. For the interactive search challenge, each of the six participants had developed an interactive search engine for the LSC collection and tested it using the six development topics. For the challenge, each participant was given a desk with a clear view of a large screen which showed the topics, the time remaining on each topic, as well as the current and overall scores of each team. When a participating team located a potentially relevant item from the collection, it was submitted to a host server which evaluated it and if it was successful, updated the team score, but if it was unsuccessful, the potential score of that team for that topic was down-weighted. 


\subsection{Overview of Participants at LSC}

The LSC 2018 [11], which was the first time that the test collection was used, attracted six participating groups. To highlight the flexibility of the collection, we report on the six different approaches to interactive retrieval taken by the six participants:

- A multi-faceted retrieval system [18], based on the video search system diveXplore [19]. Besides efficient presentation and summarisation of lifelog data, the tool includes searchable feature maps, concept and metadata filters, similarity search and sketch search.

- The LIFER retrieval system [25] that provided an efficient retrieval system based primarily on faceted querying using available metadata.

- An interactive retrieval tool [16], based on SIRET [17], that was updated to include enhanced visualisation and navigation methodologies for a high number of visually similar scenes representing repetitive daily activities.

- A Virtual Reality interactive retrieval system [7] that uses visual concepts and dates/times as the basis for a faceted filtering mechanism that presents results in a novel VR-interface.

- A clustering retrieval system [24] that groups images into visual shots and clusters, extracts semantic concepts on scene category and attributes, entities, and actions, and supports 4 main types of query conditions: temporal, spatial, entity and action, and extra data criteria.

- A faceted lifelog search mechanism [1]) that introduced a four-step process required to support lifelog search engines and provided a ranked list of items as a sequential list of item clusters, as opposed to items themselves.

Four of the systems that took part performed comparatively well, with participants finding results within the time-limit for most of the topics. However, it is worth noting that the top two performing teams [7] and [18] were very close in performance, with a very minor separation in overall performance.

\subsection{Description of the Experimental Infrastructure at LSC}

During the Lifelog Search Challenge event, a similar infrastructure to that used at the VBS was employed to coordinate the competition. A host server coordinated, the display of the temporally advancing topics, the timer for each topic, evaluated the submissions from each team in real-time, calculated the points awarded to each team for a successful submission, and displayed a live scoreboard. The points awarded for a successful submission were based on a formula that rewarded the speed of submission, but also penalised an incorrect submission. An incorrect submission would result in a $20 \%$ reduction in the total available points for that topic, where the number of available points were decreasing every second. This added an element of excitement to the live competition. 


\section{Conclusions \& Collection Availability}

In this paper, we have introduced a new test collection for interactive lifelog retrieval. To the best of our knowledge, it is the most rich multimdoal collection of lifelog / personal sensor data that has been released for comparative experimentation. The dataset extends for 27 days with data items for every minute of this time. We also introduced a new type of temporally-advancing topic for use in interactive retrieval experimentation and we reported on the types of interactive systems that were developed for this test collection and entered by participating research teams at the Lifelog Search Challenge competition at ACM ICMR 2018.

The LSC test collection (and associated documentation) is available for download from the LSC website ${ }^{6}$. Anyone using the dataset must sign two forms to access the datasets, an organisational agreement form for the organisation (signed by the research team leader) and an individual agreement form for each member of the research team that will access the data. This is requested in order to adhere to host data governance policies for lifelog data. The test collection is composed of a number of files; the core image dataset, the associated metadata, the information access dataset and the provided visual concept data for each image. Each zip file is additionally password protected.

\section{Acknowledgements}

We acknowledge the financial support of Science Foundation Ireland (SFI) under grant number SFI/12/RC/2289 and JSPS KAKENHI under Grant Number $18 \mathrm{H} 00974$.

\section{References}

1. A. Alsina, X. Giró, and C. Gurrin. An Interactive Lifelog Search Engine for LSC2018. In ACM Workshop on The Lifelog Search Challenge, LSC '18, pages 30-32, New York, NY, USA, 2018. ACM.

2. V. Bush. As we may think. interactions, 3(2):35-46, Mar. 1996.

3. D.-T. Dang-Nguyen, L. Piras, M. Riegler, G. Boato, L. Zhou, and C. Gurrin. Overview of ImageCLEFlifelog 2017: Lifelog Retrieval and Summarization. In CLEF2017 Working Notes, Dublin, Ireland, September 11-14 2017.

4. D.-T. Dang-Nguyen, L. Piras, M. Riegler, L. Zhou, M. Lux, and C. Gurrin. Overview of ImageCLEFlifelog 2018: Daily Living Understanding and Lifelog Moment Retrieval. In CLEF2018 Working Notes, CEUR Workshop Proceedings, Avignon, France, September 10-14 2018. CEUR-WS.org.

5. D.-T. Dang-Nguyen, L. Zhou, R. Gupta, M. Riegler, and C. Gurrin. Building a Disclosed Lifelog Dataset: Challenges, Principles and Processes. In Content-Based Multimedia Indexing (CBMI), 2017.

6. M. Dodge and R. Kitchin. 'Outlines of a world coming into existence': Pervasive computing and the ethics of forgetting. Environment and Planning B: Planning and Design, 34(3):431-445, 2007.

\footnotetext{
${ }^{6}$ Lifelog Search Challenge website: http://lsc.dcu.ie/. Last Visited 27th July 2018.
} 
7. A. Duane, C. Gurrin, and W. Huerst. Virtual Reality Lifelog Explorer: Lifelog Search Challenge at ACM ICMR 2018. In ACM Workshop on The Lifelog Search Challenge, LSC '18, pages 20-23, New York, NY, USA, 2018. ACM.

8. C. Gurrin, H. Joho, F. Hopfgartner, L. Zhou, and R. Albatal. NTCIR Lifelog: The First Test Collection for Lifelog Research. In Proceedings of SIGIR'16 Conference, pages 705-708. ACM, 2016.

9. C. Gurrin, H. Joho, F. Hopfgartner, L. Zhou, and R. Albatal. Overview of NTCIR12 Lifelog Task. In Proc. of the 12th NTCIR Conference, pages 354-360, 2016.

10. C. Gurrin, H. Joho, F. Hopfgartner, L. Zhou, R. Gupta, R. Albatal, and D.-T. Dang-Nguyen. Overview of NTCIR-13 Lifelog-2 Task. In Proc. of the 13th NTCIR Conference, pages 6-11, 2017.

11. C. Gurrin, K. Schoeffmann, H. Joho, D.-T. Dang-Nguyen, M. Riegler, and L. Piras, editors. LSC '18: Proceedings of the 2018 ACM Workshop on The Lifelog Search Challenge, New York, NY, USA, 2018. ACM.

12. W. Jones and J. Teevan. Personal information management. University of Washington Press, 2011.

13. M. P. Kato and Y. Liu. Overview of NTCIR-13. pages 1-5, 2017.

14. P. H. Kim and F. Giunchiglia. The Open Platform for Personal Lifelogging: The eLifeLog Architecture. In CHI '13 Extended Abstracts on Human Factors in Computing Systems, CHI EA '13, pages 1677-1682, New York, NY, USA, 2013. ACM.

15. Y. J. Lee, J. Ghosh, and K. Grauman. Discovering important people and objects for egocentric video summarization. In Computer Vision and Pattern Recognition (CVPR), 2012 IEEE Conference on, pages 1346-1353. IEEE, 2012.

16. J. Lokoc, W. Bailer, K. Schoeffmann, B. Muenzer, and G. Awad. On influential trends in interactive video retrieval: Video Browser Showdown 2015-2017. IEEE Transactions on Multimedia, 2018.

17. J. Lokoc, G. Kovalcík, and T. Soucek. Revisiting SIRET video retrieval tool. In MultiMedia Modeling - 24th International Conference, MMM 2018, Bangkok, Thailand, February 5-7, 2018, Proceedings, Part II, pages 419-424, 2018.

18. B. Münzer, A. Leibetseder, S. Kletz, M. J. Primus, and K. Schoeffmann. lifeXplore at the Lifelog Search Challenge 2018. In ACM Workshop on The Lifelog Search Challenge, LSC '18, pages 3-8, New York, NY, USA, 2018. ACM.

19. K. Schoeffmann, B. Münzer, J. Primus, and A. Leibetseder. The diveXplore System at the Video Browser Showdown 2018 - Final Notes. CoRR, abs/1804.01863, 2018.

20. A. J. Sellen and S. Whittaker. Beyond total capture. Communications of the ACM, 53(5):70, 52010.

21. A. D. Sole. Microsoft Computer Vision APIs Distilled: Getting Started with Cognitive Services. Apress, 2017.

22. S. Song, V. Chandrasekhar, N.-M. Cheung, S. Narayan, L. Li, and J.-H. Lim. Activity recognition in egocentric life-logging videos. In Asian Conference on Computer Vision, pages 445-458. Springer, 2014.

23. E. Talavera, M. Dimiccolo, M. Bolanos, M. Aghaei, and P. Radeva. R-clustering for egocentric video segmentation. In Iberian Conference on Pattern Recognition and Image Analysis, pages 327-336. Springer Verlag, 2017.

24. T.-D. Truong, T. Dinh-Duy, V.-T. Nguyen, and M.-T. Tran. Lifelogging retrieval based on semantic concepts fusion. In ACM Workshop on The Lifelog Search Challenge, LSC '18, pages 24-29, New York, NY, USA, 2018. ACM.

25. L. Zhou, Z. Hinbarji, D.-T. Dang-Nguyen, and C. Gurrin. Lifer: An interactive lifelog retrieval system. In ACM Workshop on The Lifelog Search Challenge, LSC '18, pages 9-14, New York, NY, USA, 2018. ACM. 